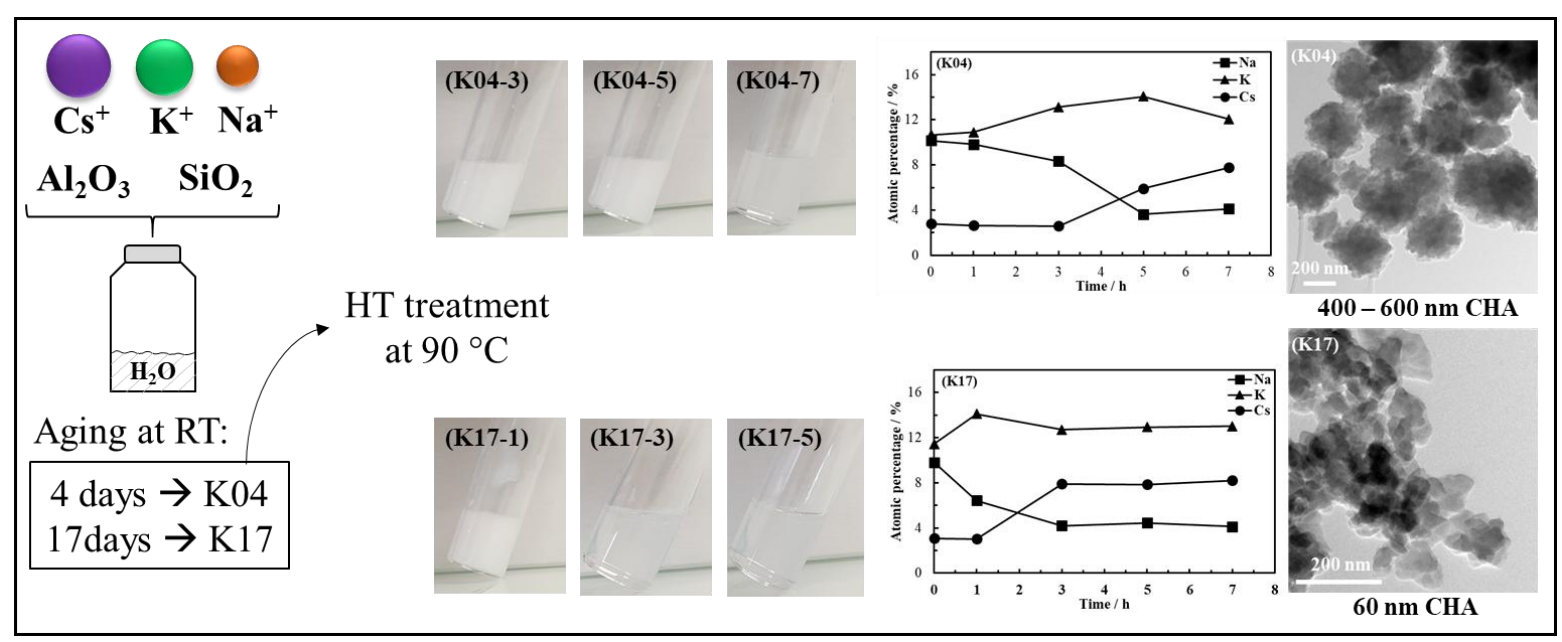




\title{
The role of mixed alkali metal cations on the formation of nanosized CHA zeolite from colloidal precursor suspension
}

\author{
Sajjad Ghojavand ${ }^{\mathrm{a}}$, Edwin B. Clatworthy ${ }^{\mathrm{a}}$, Aurélie Vicente ${ }^{\mathrm{a}}$, Eddy Dib $^{\mathrm{a}}$, Valérie Ruaux ${ }^{\mathrm{a}}$, \\ Maxime Debost $^{\mathrm{a}, \mathrm{b}}$, Jaafar El Fallah $^{\mathrm{a}}$, Svetlana Mintova ${ }^{\mathrm{a}^{*}}$ \\ ${ }^{a}$ Normandie Université, ENSICAEN, UNICAEN, CNRS, Laboratoire Catalyse et \\ Spectrochimie (LCS), 14000 Caen, France \\ ${ }^{\mathrm{b}}$ Normandie Université, ENSICAEN, UNICAEN, CNRS, CRISMAT, 14000 Caen, France
}

\begin{abstract}
:
A clear understanding of the crystal formation pathways of zeolites remains one of the most challenging issues to date. Here we investigate the synthesis of nanosized chabazite (CHA) zeolites using organic template-free colloidal suspensions by varying the time of aging at room temperature and the time of hydrothermal treatment at $90{ }^{\circ} \mathrm{C}$. The role of mixed alkali metal cations $\left(\mathrm{Na}^{+}, \mathrm{K}^{+}, \mathrm{Cs}^{+}\right)$ on the formation of $\mathrm{CHA}$ in the colloidal suspensions was studied. Increasing the aging time of the precursor colloidal suspension from 4 to 17 days resulted in faster crystallization of CHA nanocrystals $\left(3 \mathrm{~h}\right.$ instead of $7 \mathrm{~h}$ at $90{ }^{\circ} \mathrm{C}$ ) to afford significantly smaller particles $(60 \mathrm{~nm} \mathrm{vs} 600 \mathrm{~nm})$. During the crystallization a considerable change in the content of inorganic cations in the recovered solid material was observed to coincide with the formation of the CHA nanocrystals. The $\mathrm{Na}^{+}$cations were found to direct the formation of condensed and pre-shaped aluminosilicate particles in the colloidal precursor suspensions, while $\mathrm{K}^{+}$cations facilitated the formation of secondary building units (SBUs) of the CHA type framework structure such as $d 6 r$ and cha cages, and the $\mathrm{Cs}^{+}$cations promoted the long-range crystalline order facilitating the crystallization of stable zeolite nanocrystals.
\end{abstract}

\section{Keywords:}

Nanozeolite, chabazite, colloidal suspension, crystallization, organic template-free, inorganic cation templating

*Corresponding author. E-mail address: svetlana.mintova@ensicaen.fr

Tel: +33231452737 


\section{Introduction}

Zeolites play a critical role in industrial applications such as separation and catalysis due to their molecular-sized pore openings $(0.3-2 \mathrm{~nm})$ and shape selectivity.[1-4] Zeolites consist of ordered corner-sharing $\mathrm{TO}_{4}$ tetrahedra (typically $\mathrm{T}=\mathrm{Si}, \mathrm{Al}$ ) which can be arranged to produce a variety of different frameworks allowing for the tuning of their chemical and physical properties.[5,6] The particle size of conventional zeolites are typically in the order of micrometers and are composed of aggregates of several individual crystals.[7] Due to their size one of the major drawbacks of conventional zeolites is diffusion limitations, however, this can be addressed by decreasing the zeolite particle size.[8] Nanosized zeolites have dimensions of less than $200 \mathrm{~nm}$ and unlike conventional zeolites are typically composed of discrete particles (single crystals), exhibit greater external surface area, and possess a greater number of available active sites.[5,7,9]

There are several methods for synthesizing nanozeolites including from clear suspensions,[10,11] using crystal growth inhibitors,[12] confined space synthesis,[13,14] and from organic solvents.[15,16] Features shared across these synthesis methods include the use of a basic synthesis media, an organic structure-directing agent (OSDA), and amorphous sources of silica and alumina. The formation of nanozeolites is typically achieved by heating the reaction mixture in a sealed reactor.[9] However, nanozeolites synthesized using OSDAs have a relatively high cost, toxicity due to the presence of organic templates, and require a post-synthetic calcination that can cause partial collapse of the zeolite structure and necessitates additional energy and release of $\mathrm{CO}_{2}$.[5] A promising alternative for the synthesis of nanozeolites is the organic template-free method.[17] In this method the ionic interactions of cations with $\mathrm{SiO}_{4}{ }^{4-}$ and $\mathrm{AlO}_{4}{ }^{5-}$ tetrahedra are used to direct the formation of the nanozeolites.[5] In addition, the nanozeolites are prepared at relatively low temperatures $\left(\right.$ e.g. $\left.<100{ }^{\circ} \mathrm{C}\right)$ to favor the uniform nucleation of particles with a similar size.[5] By careful tuning of the type and content of cations, aging time, and hydrothermal temperature, various zeolites with nanoscale dimensions have been synthesized without the use of OSDAs.[3,17-19] However, key aspects of the organic template-free method such as the crystal formation and the role of different cations in directing the zeolite structure require further investigation.

We recently reported the synthesis of nanosized chabazite (CHA) using a highly alkaline mixture containing three monovalent alkali metal cations as templates $\left(\mathrm{Na}^{+}, \mathrm{K}^{+}\right.$, and $\mathrm{Cs}^{+}$).[20] The CHA framework is described by a three-dimensional pore system containing 
super cages of $6.7 \times 10 \AA$ which are accessed by eight-membered rings (8MRs) of $3.8 \times 3.8$ A..[21] Here, we describe the crystal formation in two series of samples from colloidal precursor suspensions by varying the aging time and the synthesis time resulting in nanosized CHA zeolites with different particle sizes $(60 \mathrm{~nm}$ and $600 \mathrm{~nm})$. The role of the mixed inorganic cations in directing the CHA structure was identified using a combination of ICP-MS and EDX analyses.

\section{Experimental}

\subsection{Materials and Synthesis}

All reagents were used as received unless otherwise specified. Sodium hydroxide pellets (98 wt\%), potassium hydroxide (85 wt\%), LUDOX AS-40, and sodium aluminate $\left(\mathrm{NaAlO}_{2}, 40-45 \% \mathrm{Na}_{2} \mathrm{O}, 50-56 \% \mathrm{Al}_{2} \mathrm{O}_{3}\right)$ were purchased from Sigma-Aldrich. Cesium hydroxide hydrate (15-20 wt $\left.\% \mathrm{H}_{2} \mathrm{O}\right)$ was purchased from Alfa Aesar. Double distilled (dd) water was used for all syntheses. Synthesis of the CHA samples was based on a modified protocol published recently by our group.[20] $0.54 \mathrm{~g}$ of $\mathrm{NaAlO}_{2}$ was mixed with $4.4 \mathrm{~g}$ of water under rapid stirring. After obtaining a clear suspension, $1.7 \mathrm{~g}$ of $\mathrm{NaOH}, 0.824 \mathrm{~g}$ of $\mathrm{KOH}$, and $0.217 \mathrm{~g}$ of $\mathrm{CsOH}(50 \mathrm{wt} \% \mathrm{Cs}$ in water) were added and stirred for 2 hours to obtain a clear solution. Afterwards, $10 \mathrm{~g}$ of LUDOX AS-40 was added dropwise under rapid stirring. The final colloidal suspension precursor composition was $0.2 \mathrm{Cs}_{2} \mathrm{O}: 1.5 \mathrm{~K}_{2} \mathrm{O}$ : $6.0 \mathrm{Na}_{2} \mathrm{O}: 16.0 \mathrm{SiO}_{2}: 0.7 \mathrm{Al}_{2} \mathrm{O}_{3}: 141.7 \mathrm{H}_{2} \mathrm{O}$. Two series of samples were prepared whereby the colloidal precursor suspensions were aged at room temperature with vigorous stirring for either 4 days (K04 series) or 17 days (K17 series). Hydrothermal (HT) treatment of the colloidal precursor suspensions was performed in a static oven at $90{ }^{\circ} \mathrm{C}$ for up to $9 \mathrm{~h}$. After HT treatment the solid material was recovered by centrifugation and washed with hot water $\left(90{ }^{\circ} \mathrm{C}\right)$ until neutral $\mathrm{pH}(7-8)$ of the decanted solution was achieved. The recovered material was dried in an oven at $60{ }^{\circ} \mathrm{C}$ overnight. The samples obtained from the series K04 after $1,3,5,7$, and $9 \mathrm{~h}$ of HT treatment are abbreviated as K04-1, -3, -5, -7, and -9, while the samples from series K17 after 1, 3, 5, and $7 \mathrm{~h}$ of HT treatment are abbreviated as K17-1, $-3,-5$, and -7 .

\subsection{Sample Characterization}

Powder X-ray diffraction (XRD) patterns of zeolites were collected with a PANalytical X'Pert Pro diffractometer equipped with a Johansson monochromator using the 
$\mathrm{Cu}$ Kal radiation $(\lambda=1.540598 \AA$ ). Scanning electron microscopy (SEM) of samples was performed using a Tescan Mira I LMH under $20 \mathrm{kV}$. Energy dispersive X-ray (EDX) analysis of samples was performed using two Bruker XFLASH 6/30 EDX cameras. The crystal size and morphology of solids were determined by high-resolution transmission electron microscopy (HR-TEM) using a FEI LaB 6 TECNAI G2 $30 \mathrm{UT}$ operated at $300 \mathrm{kV}$. Viscosity measurements of the colloidal suspension of series K04 and K17 were performed using a rotational rheometer from Kenexus and a PU20 SC0085 SS geometry.

Magic-angle spinning nuclear magnetic resonance (MAS NMR) spectra of ${ }^{27} \mathrm{Al},{ }^{29} \mathrm{Si}$,

${ }^{23} \mathrm{Na}$ and ${ }^{133} \mathrm{Cs}$ nuclei were recorded with a single pulse on a Bruker Avance $400 \mathrm{MHz}(9.4$ T) spectrometer using $4 \mathrm{~mm}-\mathrm{OD}$ zirconia rotors with a spinning frequency of between 12 and $14 \mathrm{kHz}$. Details are listed in Table S2.

The FTIR spectra of samples were recorded with a Nexus IR spectrometer (1300-450 $\mathrm{cm}^{-1}$, resolution of $1 \mathrm{~cm}^{-1}, 132$ scans) using the $\mathrm{KBr}$ pellet technique. Inductively coupled plasma mass spectrometry (ICP-MS) measurements were recorded using a 7900 ICP-MS from Agilent Technologies.

\section{Results and Discussion}

\subsection{Macroscopic Characterization of Nanosized CHA Zeolites}

The XRD patterns of the samples from the K04 and K17 series are presented in Figure 1. For the K04-5 sample, the XRD pattern reveals the emergence of the diffraction peaks associated with the CHA framework structure indicating that the formation of the CHA structure begins after approximately $3 \mathrm{~h}$ of HT treatment of the colloidal precursor suspension at $90{ }^{\circ} \mathrm{C}$. Even after $5 \mathrm{~h}$ of HT treatment the diffraction peaks are broad and display low intensity signifying that a large amount of amorphous precursor material is still present. Increasing the time of HT treatment showed that fully crystalline CHA was obtained after $7 \mathrm{~h}$; no further changes were observed in the XRD patterns at longer HT treatment times (Figure S1). In comparison, for the K17 series the CHA structure is formed after $3 \mathrm{~h}$ of HT treatment showing that increasing of the aging time of the colloidal suspension leads to faster crystallization of the CHA zeolite. The fully crystalline CHA zeolite is formed more rapidly in the K17 series as evidenced by the identical XRD patterns for samples K17- 3, -5 and -7 (Figure S2). 
Microscopic analysis of CHA samples from the K04 and K17 series revealed clear morphological differences between them as a result of the different aging times. Figure 2 presents SEM images of the K04 and K17 samples showing the transformation from amorphous matter to crystalline CHA zeolite over the $7 \mathrm{~h}$ of HT treatment. Sample K04-3 (Figure 2a) consists of micron-sized amorphous agglomerated aluminosilicate particles while after $5 \mathrm{~h}$ of HT treatment crystalline CHA particles can be seen together with amorphous material (Figure 2b). The CHA particles have an average size of $\approx 600 \mathrm{~nm}$ in diameter while the amorphous material exhibits micrometer sized particles. After $7 \mathrm{~h}$ of HT treatment all of the amorphous material converts to crystalline CHA particles $\approx 600 \mathrm{~nm}$ in diameter with morphology defined by rough spheres of smaller intergrown crystallites (Figure 2c). For the K17 series, sample K17-1 shows similar amorphous appearance to sample K04-3 (Figure 2d). However, sample K17-3 consists of fully crystalline nanosized CHA crystals $60 \mathrm{~nm}$ across (Figure 2e). This is consistent with the appearance of the Bragg peaks of the CHA structure after $3 \mathrm{~h}$ of HT treatment of the colloidal precursor suspension presented in Figure 1b. Increasing the HT treatment beyond $3 \mathrm{~h}$ does not affect the size or morphology of the CHA nanocrystals (K17-5, Figure 2f), which is consistent with the XRD results (Figure 1).

TEM analysis was also performed on the fully crystalline samples K04-7 and K17-3 (Figure 3). The TEM image for the sample K04-7 clearly shows that the particles are primarily between 400 to $600 \mathrm{~nm}$ in size and are composed of smaller polycrystalline intergrown crystallites (Figure 3a), consistent with the SEM results. Smaller particles $(<200$ $\mathrm{nm}$ ) with similar morphology were also observed. In comparison, TEM analysis of the sample K17-3 shows discrete nanosized CHA crystals with sizes of less than $60 \mathrm{~nm}$ (Figure 3b) significantly smaller than the CHA reported in our previous work ( 200 nm).[20]

Rheometric analysis of the CHA samples from the K04 and K17 series are presented in Figure 4 showing the changes in the viscosity of the colloidal precursor suspension during the HT treatment. As shown in Figure 4a for sample K04-1, the viscosity of the colloidal suspension increases substantially from $2 \mathrm{cPs}$ to $50000 \mathrm{cPs}$ and remains high until $3 \mathrm{~h}$ of HT treatment due to the formation of micron-sized amorphous agglomerated aluminosilicate particles (Figure 2a). Only after $5 \mathrm{~h}$ of HT treatment does the viscosity value decrease (from $46000 \mathrm{cPs}$ to $7500 \mathrm{cPs}$ ) due to the transformation of the large amorphous agglomerates to smaller CHA crystals (Figure 1a and Figure 2b). Increasing the HT treatment to $7 \mathrm{~h}$ transforms all of the micron-sized amorphous agglomerates into crystalline CHA particles (shown in Figure 2c) and the viscosity decreases significantly from $7500 \mathrm{cPs}$ to $0.9 \mathrm{Cps}$. In 
comparison, the viscosity of the K17 series changes over a shorter period of time. Sample K17-1 shows a high viscosity similar to K04-3 followed by a significant reduction in viscosity after $3 \mathrm{~h}$ of HT treatment due to the transformation of the colloidal precursor suspension into crystalline nanosized CHA (Figure 1b, Figure 2e, and Figure 3b). The significant changes (increase followed by decrease) in viscosity of the colloidal suspension during the crystallization process can be attributed to the polymerization of the cationcoordinated $\mathrm{SiO}_{4}{ }^{4-}$ and $\mathrm{AlO}_{4}{ }^{5-}$ tetrahedra followed by the establishment of long-range crystallinity facilitated by the dynamic changes in the cation content between the solid and liquid phases in the colloidal suspension (vide infra).

\subsection{Spectroscopic Characterization of Nanosized CHA Zeolites}

To further understand the evolution of the colloidal precursor suspensions and the formation of the CHA zeolite during the crystallization process, the two series of samples were analyzed by FTIR and NMR spectroscopy. The FTIR spectra of the K04 and K17 series of samples in the range of $450-1300 \mathrm{~cm}^{-1}$ are presented in Figure 5. The formation of the secondary building units (SBUs) relative to the CHA zeolite during the crystallization process was studied. A broad vibration band related to the asymmetric stretching of $\mathrm{Si}-\mathrm{O}-\mathrm{T}$ ( $\mathrm{T}=\mathrm{Al}$ or $\mathrm{Si}$ ) can be seen at $950-1200 \mathrm{~cm}^{-1}$ for all samples,[22,23] while the symmetric vibration of $\mathrm{Si}-\mathrm{O}-\mathrm{T}$ also appears at $670-820 \mathrm{~cm}^{-1} \cdot[24,25]$ This suggests that prior to the detection of the long-range crystalline order in the CHA zeolite observable by XRD, polymerization of silica and alumina resulting in the formation of amorphous aluminosilicates possessing short range crystalline order (i.e. crystal nuclei) is observed by FTIR spectroscopy. In the K04 series, the sample K04-5 contains a peak at $466 \mathrm{~cm}^{-1}$ corresponding to the $\mathrm{O}-\mathrm{Si}-\mathrm{O}$ bending mode.[26,27] After $7 \mathrm{~h}$ two new bands at 518 and $635 \mathrm{~cm}^{-1}$ appeared which can be attributed to double six-membered rings $(d 6 r)$ and the 8MR as a secondary building unit (SBU) in the cha cage, respectively. In comparison, in the spectrum of sample $\mathrm{K} 17-3$ the shoulder at $466 \mathrm{~cm}^{-1}$ attributed to the $\mathrm{O}-\mathrm{Si}-\mathrm{O}$ bending mode is already present. Both the $d 6 r$ and $8 \mathrm{MR}$ bands also appear in the spectrum of sample K173 and remain relatively unchanged with further HT treatment of the colloidal suspensions (Figure S3). These results suggest that the crystallization of the CHA zeolite starts with the formation of $d 6 r$ and cha structures and that their further development into a long-range crystalline structure requires a template such as an appropriate organic or inorganic cation, or a mixture of both. 
${ }^{27} \mathrm{Al}$ MAS NMR analysis of the K04 and K17 series were performed and the spectra are presented in Figure 6a,b. All samples in these series show a resonance at 57 ppm which is related to the tetrahedrally coordinated $\mathrm{Al}$ atoms, and no signal corresponding to extraframework $\mathrm{Al}$ is observed. This agrees with the appearance of $\mathrm{Si}-\mathrm{O}-\mathrm{T}(\mathrm{T}=\mathrm{Al}$ or $\mathrm{Si})$ stretching and vibrational bands in the FTIR spectra presented in Figure 5, suggesting the formation of a polymerized aluminosilicate amorphous or crystalline (depending on the HT treatment time) species after aging of the samples. For the K04 series (Figure 6a), sample K04-3 shows a broad asymmetric resonance which suggests a heterogeneous surrounding of Al typical of amorphous aluminosilicates which is consistent with XRD pattern presented in Figure 1a. Increasing the HT treatment to $5 \mathrm{~h}$ sharpens the resonance while $7 \mathrm{~h}$ of HT treatment reveals a more intense sharp symmetric resonance related to the fully crystalline tetrahedral Al. For the K17 series (Figure 6b) the sample K17-1 shows an asymmetric resonance in the ${ }^{27} \mathrm{Al}$ spectrum related to the amorphous aluminosilicates observed similarly in sample K04-3. However, after $3 \mathrm{~h}$ of HT treatment the ${ }^{27} \mathrm{Al}$ spectrum reveals a symmetric resonance at $57 \mathrm{ppm}$ typical of crystalline structures. The band at $57 \mathrm{ppm}$ does not change with increasing the HT treatment time to $5 \mathrm{~h}$. These results are consistent with the XRD, electron microscopy and FTIR analyses.

${ }^{29} \mathrm{Si}$ MAS NMR analysis for the K04 and K17 series are presented in Figure 6c,d. Samples K04-3 and K04-5 show asymmetric broad resonances from -110 ppm to $-80 \mathrm{ppm}$ which suggests a complex heterogeneous tetrahedrally coordinated $\mathrm{Si}$ atom in amorphous aluminosilicates (Figure 6c). In comparison, sample K04-7 displays four resonances at -94, 99, -104, and $-110 \mathrm{ppm}$ correspond to $3 \mathrm{Al}\left(\mathrm{Si}[\mathrm{SiO}][\mathrm{AlO}]_{3}\right), 2 \mathrm{Al}\left(\mathrm{Si}[\mathrm{SiO}]_{2}[\mathrm{AlO}]_{2}\right), 1 \mathrm{Al}$ $\left(\mathrm{Si}[\mathrm{SiO}]_{3}[\mathrm{AlO}]\right)$, and $0 \mathrm{Al}\left(\mathrm{Si}\left[\mathrm{SiO}_{4}\right)\right.$ species.[20] For the $\mathrm{K} 17$ series (Figure 6d), sample K17-1 shows similar results to the samples K04-3 and K04-5 while for samples K17-3 and K17-5 the different crystallographic Si environments can be observed and are similar to those of sample K04-7. The increased HT treatment from $3 \mathrm{~h}$ to $5 \mathrm{~h}$ does not result in any changes in the ${ }^{29} \mathrm{Si}$ NMR spectra for the K17 series, similar to the results shown for XRD and FTIR, thus confirming that fully crystalline CHA was obtained after $3 \mathrm{~h}$. The Si/Al ratio of the fully crystalline samples K04-7 and K17-3 calculated by the Engelhardt and Michel equation is 2.1 (Table 1, S1). For comparison the Si/Al ratio of the samples obtained from ICP-MS and EDX are presented.[28] As shown, the Si/Al ratio of the fully crystalline samples (K04-7 and K17-3) is similar, which suggests a similar amount of alkali metal cations are present. 
To study the placement and migration of the extra-framework cations ${ }^{133} \mathrm{Cs}$ MAS NMR analyses were performed on amorphous, semicrystalline, and crystalline samples from both series of CHA zeolites synthesized from the colloidal suspensions (Figure S4). ${ }^{133} \mathrm{Cs}$ MAS NMR spectra reveal a symmetric resonance at $-61 \mathrm{ppm}$ in samples K04-5, K04-7, $\mathrm{K} 17-3$, and K17-5. This is assigned to a single $\mathrm{Cs}^{+}$extra-framework site located in the middle of the 8MR opening of the CHA structure, consistent with our previous results.[20] A separate study dedicated to understanding the exact location and dynamics of the $\mathrm{Na}^{+}$ cations using ${ }^{23} \mathrm{Na}$ MQMAS NMR and Rietveld analyses will be presented in future work.

In addition to determining the $\mathrm{Si} / \mathrm{Al}$ ratio, the content of the extra-framework cations used to template the CHA structure was measured by ICP-MS and EDX. The atomic percentage of extra-framework cations $\left(\mathrm{Na}^{+}, \mathrm{K}^{+}, \mathrm{Cs}^{+}\right)$in the recovered solid material of the K04 and K17 series based on ICP-MS is presented in Figure 7. For the K04 series (Figure 7a), the $\mathrm{Na}^{+}$content decreases consistently until $5 \mathrm{~h}$, coincident with an increase in the $\mathrm{K}^{+}$ cation content to its maximum value. The $\mathrm{Cs}^{+}$content begins increasing after approximately $3 \mathrm{~h}$ of HT treatment at which time the crystallization of the CHA structure begins. Beyond 5 $\mathrm{h}$ the $\mathrm{K}^{+}$content decreases slightly, coincident with a continued increase in the $\mathrm{Cs}^{+}$content and further crystallization of the CHA until $7 \mathrm{~h}$. These results suggest that the high $\mathrm{Na}^{+}$ content is required to stabilize the colloidal precursor suspension containing amorphous nanosized particles. The $\mathrm{K}^{+}$cations direct the formation of SBUs such as the $d 6 r$ s and 8MRs of the CHA type framework. After the formation of the SBUs in sample K04-5, the first CHA crystals are observed (see Figure $2 \mathrm{~b}$, Figure 5a). During the crystallization, the $\mathrm{Cs}^{+}$ partially replaces the $\mathrm{Na}^{+}$and $\mathrm{K}^{+}$cations leading to the formation of long-range crystalline matter, where a sufficient connectivity between the SBUs is achieved leading to the formation of fully crystalline CHA zeolite crystals (sample K04-7), see Figure 1a and Figure 7a. The same trend in the K17 series is observed. The changes in the cation content in the K17 series are corroborated with the macroscopic and spectroscopic results (Figure 1b and Figure 5b). The ICP-MS results are in good agreement with the EDX data (Supplementary Information, Figure S5).

The role of the mixed inorganic cations used for the synthesis of CHA zeolite is presented schematically in Scheme S1. Initially, a colloidal precursor suspension containing a high concentration of inorganic cations $\left(\mathrm{Na}^{+}, \mathrm{K}^{+}\right.$and $\left.\mathrm{Cs}^{+}\right)$was stabilized at room temperature and subjected to aging at RT for 4 days (K04) and 17 days (K017). After 4 days of aging (K04 series), the colloidal precursor mixture contains large micron-sized amorphous aluminosilicates with a broad size distribution (Figure 2a). After subjecting this 
colloidal precursor mixture to $5 \mathrm{~h}$ of $\mathrm{HT}$ treatment the content of $\mathrm{K}^{+}$and $\mathrm{Cs}^{+}$in the samples increases while the $\mathrm{Na}^{+}$decreases (Figure $7 \mathrm{a}$ ). $\mathrm{K}^{+}$cations promote the formation of different structures $(d 6 r$ and $8 \mathrm{MR})$, and due to the presence of $\mathrm{Cs}^{+}$cations the crystallization of the CHA type zeolite is accomplished. Increasing the HT treatment to $7 \mathrm{~h}$ (K04-7) induces further crystal growth resulting in CHA zeolite with a particle size of $600 \mathrm{~nm}$. The CHA polycrystalline particles are composed of intergrown nanosized CHA crystals (Figure 3a). In comparison, after 17 days of aging (K17 series) the colloidal precursor suspension contains smaller amorphous aluminosilicate particles in comparison to the K04 series (Figure 2a,d). After $3 \mathrm{~h}$ of HT treatment (the amorphous aluminosilicate particles transform into discrete nanosized CHA crystals with a size of less than $60 \mathrm{~nm}$ (Figure 3b). The change in content of alkali metal cations in the recovered solid material is similar to the K04 series; a complete transformation from amorphous into crystalline particles is achieved within $3 \mathrm{~h}$ instead of 7 h compared to the K04 series (Figure 1b and Figure 7b). While the content of the alkali metal cations changes considerably during the HT treatment of both series. These results clearly highlight the important role of the inorganic cations in the synthesis of CHA zeolite nanocrystals.

\section{Conclusions}

The crystallization of nanosized CHA zeolite from colloidal precursor suspensions containing high concentration of mixed inorganic structure-directing cations $\left(\mathrm{Na}^{+}, \mathrm{K}^{+}\right.$and $\mathrm{Cs}^{+}$) subjected to room temperature aging for 4 and 17 days is reported. The crystallization kinetics, size, and morphology of the CHA crystals strongly depends on the aging time of the colloidal precursor suspensions. Four days of aging of the colloidal precursor suspension (K04 series) results in the formation of CHA zeolite composed of intergrown nanocrystals with a final particle size of $600 \mathrm{~nm}\left(7 \mathrm{~h} \mathrm{HT}\right.$ treatment at $\left.90{ }^{\circ} \mathrm{C}\right)$. In comparison, it is assumed that the aging of the same colloidal precursor suspension for seventeen days (K17 series) resulted in the formation of monodispersed amorphous nanoparticles that transformed into CHA nanocrystals with a size of $60 \mathrm{~nm}\left(3 \mathrm{~h} \mathrm{HT}\right.$ treatment at $\left.90{ }^{\circ} \mathrm{C}\right)$. The high concentration of mixed inorganic cations $\left(\mathrm{Na}^{+}, \mathrm{K}^{+}\right.$, and $\left.\mathrm{Cs}^{+}\right)$is required to (i) solubilize the $\mathrm{Si}$ and facilitate stable colloidal suspensions containing amorphous particles enriched in $\mathrm{Na}^{+}$, (ii) direct the formation of SBUs $(d 6 r, 8 \mathrm{MR})$ of the CHA type framework structure during the hydrothermal treatment by the high amount of $\mathrm{K}^{+}$, and (iii) promote the formation of long-range crystalline order resulting in the crystallization of chabazite zeolite 
containing $\mathrm{Cs}^{+}$extra-framework cations. In combination with the factors of aging and HT treatment, understanding the role of inorganic cations in directing the formation of structural units and the crystallization of framework structures is critical to the development of intelligently designed porous materials with exceptional performance.

\section{Acknowledgement}

Financial support from the Normandy Region through the RIN Recherche Program (grant 18P01675) and the Industrial Chair ANR-TOTAL "Nanoclean Energy" (Grant IPA 5621) is acknowledged. assistance of Dr. Cassandre Kouvatas in obtaining NMR data is also appreciated.

\section{References}

[1] J. Shang, G. Li, R. Singh, Q. Gu, K.M. Nairn, T.J. Bastow, N. Medhekar, C.M. Doherty, A.J. Hill, J.Z. Liu, P.A. Webley, Discriminative Separation of Gases by a "Molecular Trapdoor" Mechanism in Chabazite Zeolites, J. Am. Chem. Soc. 134 (2012) 19246-19253. https://doi.org/10.1021/ja309274y.

[2] S.V. Konnov, F. Dubray, E.B. Clatworthy, C. Kouvatas, J.-P. Gilson, J.-P. Dath, D. Minoux, C. Aquino, V. Valtchev, S. Moldovan, S. Koneti, N. Nesterenko, S. Mintova, Novel Strategy for the Synthesis of Ultra-Stable Single-Site Mo-ZSM-5 Zeolite Nanocrystals, Angew. Chem. Int. Ed. 59 (2020). https://doi.org/10.1002/anie.202006524.

[3] J. Grand, N. Barrier, M. Debost, E.B. Clatworthy, F. Laine, P. Boullay, N. Nesterenko, J.-P. Dath, J.-P. Gilson, S. Mintova, Flexible Template-Free RHO Nanosized Zeolite for Selective $\mathrm{CO}_{2} \quad$ Adsorption, Chem. Mater. 32 (2020) 5985-5993. https://doi.org/10.1021/acs.chemmater.0c01016.

[4] E.B. Clatworthy, S.V. Konnov, F. Dubray, N. Nesterenko, J.-P. Gilson, S. Mintova, Emphasis on the properties of metal-containing zeolites operating outside the comfort zone of current heterogeneous catalytic reactions, Angew. Chem. Int. Ed. 59 (2020). https://doi.org/10.1002/anie.202005498.

[5] S. Mintova, J.-P. Gilson, V. Valtchev, Advances in nanosized zeolites, Nanoscale. 5 (2013) 6693-6703. https://doi.org/10.1039/C3NR01629C.

[6] E. Koohsaryan, M. Anbia, Nanosized and hierarchical zeolites: A short review, Chin. J. Catal. 37 (2016) 447-467. https://doi.org/10.1016/S1872-2067(15)61038-5.

[7] L. Tosheva, V.P. Valtchev, Nanozeolites: Synthesis, Crystallization Mechanism, and Applications, Chem. Mater. 17 (2005) 2494-2513. https://doi.org/10.1021/cm047908z.

[8] S. Mintova, J. Grand, V. Valtchev, Nanosized zeolites: Quo Vadis?, Comptes Rendus Chim. 19 (2016) 183-191. https://doi.org/10.1016/j.crci.2015.11.005.

[9] G.-T. Vuong, T.-O. Do, Nanozeolites and Nanoporous Zeolitic Composites: Synthesis and Applications, in: Mesoporous Zeolites, John Wiley \& Sons, Ltd, 2015: pp. 79-114. https://doi.org/10.1002/9783527673957.ch3.

[10] J.P. Verduijn, Nanometer-sized molecular sieve crystals or agglomerates and processes for their production, US7767192B1, 2010. https://patents.google.com/patent/US7767192B1/en (accessed August 21, 2020).

[11] B.J. Schoeman, J. Sterte, J.-E. Otterstedt, Colloidal zeolite suspensions, Zeolites. 14 (1994) 110-116. https://doi.org/10.1016/0144-2449(94)90004-3. 
[12] H. Hosokawa, K. Oki, Synthesis of Nanosized A-type Zeolites from Sodium Silicates and Sodium Aluminates in the Presence of a Crystallization Inhibitor, Chem. Lett. 32 (2003) 586587. https://doi.org/10.1246/cl.2003.586.

[13] I. Schmidt, C. Madsen, C.J.H. Jacobsen, Confined Space Synthesis. A Novel Route to Nanosized Zeolites, Inorg. Chem. 39 (2000) 2279-2283. https://doi.org/10.1021/ic991280q.

[14] I. Schmidt, A. Krogh, K. Wienberg, A. Carlsson, M. Brorson, C.J.H. Jacobsen, Catalytic epoxidation of alkenes with hydrogen peroxide over first mesoporous titanium-containing zeolite, Chem. Commun. (2000) 2157-2158. https://doi.org/10.1039/B006460M.

[15] G.-T. Vuong, V.-T. Hoang, D.-T. Nguyen, T.-O. Do, Synthesis of nanozeolites and nanozeolite-based FCC catalysts, and their catalytic activity in gas oil cracking reaction, Appl. Catal. Gen. 382 (2010) 231-239. https://doi.org/10.1016/j.apcata.2010.04.049.

[16] G.-T. Vuong, T.-O. Do, Synthesis of silylated nanozeolites in the presence of organic phase: Two-phase and single-phase methods, Microporous Mesoporous Mater. 120 (2009) 310-316. https://doi.org/10.1016/j.micromeso.2008.11.029.

[17] H. Awala, J.-P. Gilson, R. Retoux, P. Boullay, J.-M. Goupil, V. Valtchev, S. Mintova, Template-free nanosized faujasite-type zeolites, Nat. Mater. 14 (2015) 447-451. https://doi.org/10.1038/nmat4173.

[18] V.P. Valtchev, L. Tosheva, K.N. Bozhilov, Synthesis of Zeolite Nanocrystals at Room Temperature, Langmuir. 21 (2005) 10724-10729. https://doi.org/10.1021/la050323e.

[19] E.B. Clatworthy, M. Debost, N. Barrier, S. Gascoin, P. Boullay, A. Vicente, J.-P. Gilson, J.-P. Dath, N. Nesterenko, S. Mintova, Room-Temperature Synthesis of BPH Zeolite Nanosheets Free of Organic Template with Enhanced Stability for Gas Separations, ACS Appl. Nano Mater. 4 (2021) 24-28. https://doi.org/10.1021/acsanm.0c02925.

[20] M. Debost, P.B. Klar, N. Barrier, E.B. Clatworthy, J. Grand, F. Lainé, P. Brazda, L. Palatinus, N. Nesterenko, P. Boullay, S. Mintova, Synthesis of discrete CHA zeolite nanocrystals without organic templates for selective $\mathrm{CO}_{2}$ capture, Angew. Chem. 59 (2020). https://doi.org/10.1002/ange.202009397.

[21] J. Shang, G. Li, R. Singh, P. Xiao, J.Z. Liu, P.A. Webley, Determination of Composition Range for "Molecular Trapdoor" Effect in Chabazite Zeolite, J. Phys. Chem. C. 117 (2013) 12841-12847. https://doi.org/10.1021/jp4015146.

[22] H. Sanaeishoar, M. Sabbaghan, F. Mohave, Synthesis and characterization of micromesoporous MCM-41 using various ionic liquids as co-templates, Microporous Mesoporous Mater. 217 (2015) 219-224. https://doi.org/10.1016/j.micromeso.2015.06.027.

[23] Y.-K. Ma, S. Rigolet, L. Michelin, J.-L. Paillaud, S. Mintova, F. Khoerunnisa, T.J. Daou, E.-P. $\mathrm{Ng}$, Facile and fast determination of $\mathrm{Si} / \mathrm{Al}$ ratio of zeolites using FTIR spectroscopy technique, $\begin{array}{lllll}\text { Microporous } & \text { Mesoporous } & \text { Mater. } & 311 & \text { (2021) }\end{array}$ https://doi.org/10.1016/j.micromeso.2020.110683.

[24] P. Morales-Pacheco, F. Alvarez, L. Bucio, J.M. Domínguez, Synthesis and Structural Properties of Zeolitic Nanocrystals II: FAU-Type Zeolites, J. Phys. Chem. C. 113 (2009) 2247-2255. https://doi.org/10.1021/jp8070713.

[25] M. Handke, M. Kwaśny, Infrared spectroscopic study of octahydridooctasilsesquioxane hydrolytic polycondensation, Vib. Spectrosc. 74 (2014) 127-131. https://doi.org/10.1016/j.vibspec.2014.05.007.

[26] E.M. Flanigen, H. Khatami, H.A. Szymanski, Infrared Structural Studies of Zeolite Frameworks, in: Mol. Sieve Zeolites-I, American Chemical Society, 1974: pp. 201-229. https://doi.org/10.1021/ba-1971-0101.ch016.

[27] X. Guo, D.R. Corbin, A. Navrotsky, Thermodynamics of $\mathrm{H}_{2} \mathrm{O}$ and $\mathrm{CO}_{2}$ Absorption and GuestInduced Phase Transitions in Zeolite RHO, J. Phys. Chem. C. 122 (2018) 20366-20376. https://doi.org/10.1021/acs.jpcc.8b06070.

[28] G. Engelhardt, D. Michel, High-resolution solid-state NMR of silicates and zeolites, (1987). https://www.osti.gov/biblio/6743230 (accessed July 23, 2020).

[29] Z. Zhao, Y. Xing, S. Li, X. Meng, F. Xiao, R. McGuire, A.-N. Parvulescu, U. Müller, W. Zhang, Mapping Al Distributions in SSZ-13 Zeolites from ${ }^{23} \mathrm{Na}$ Solid-State NMR Spectroscopy and DFT Calculations, J. Phys. Chem. C. 122 (2018) 9973-9979. https://doi.org/10.1021/acs.jpcc.8b01423. 
[30] L.J. Smith, H. Eckert, A.K. Cheetham, Potassium Cation Effects on Site Preferences in the Mixed Cation Zeolite Li,Na-Chabazite, Chem. Mater. 13 (2001) 385-391. https://doi.org/10.1021/cm0006392.

[31] L.J. Smith, H. Eckert, A.K. Cheetham, Site Preferences in the Mixed Cation Zeolite, Li,NaChabazite: A Combined Solid-State NMR and Neutron Diffraction Study, J. Am. Chem. Soc. 122 (2000) 1700-1708. https://doi.org/10.1021/ja992882b. 

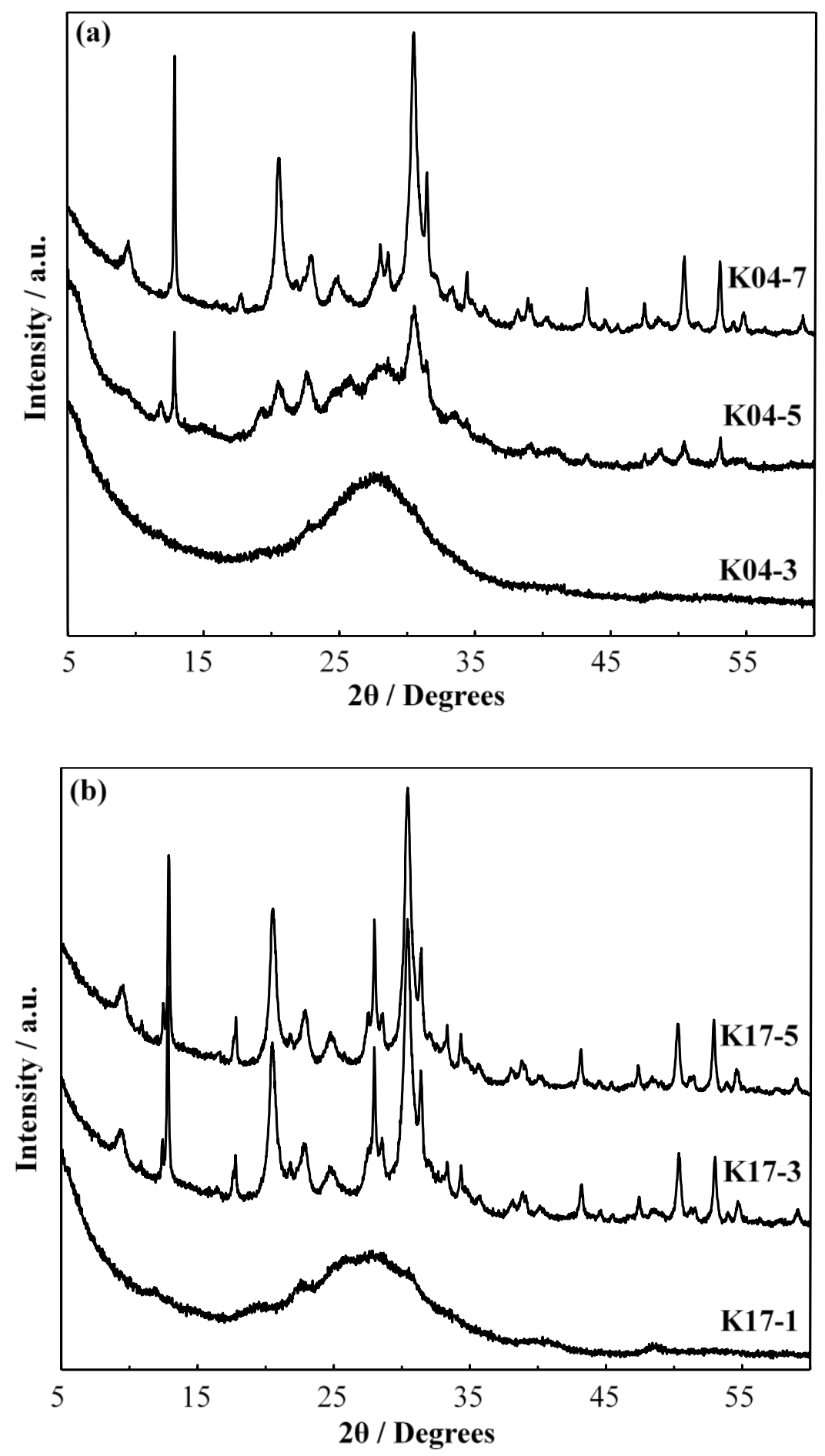

Figure 1. XRD patterns of the nanosized CHA samples (a) K04-3, K04-5, and K04-7 (b) K17-1, K17-03, and K17-5 synthesized from colloidal precursor suspensions aged four and seventeen days, respectively. 

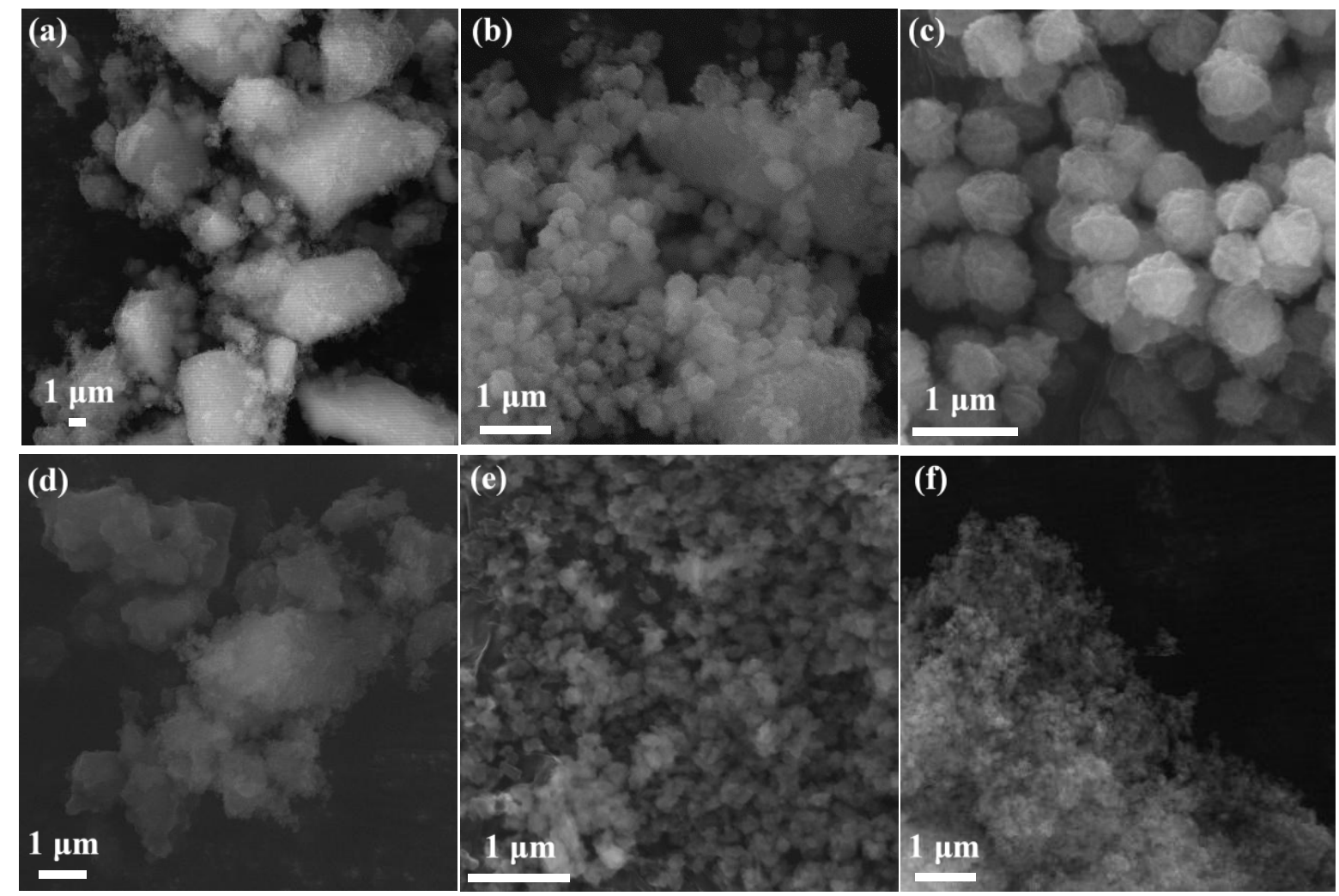

Figure 2. SEM images of the nanosized CHA samples (a) K04-3, (b) K04-5, and (c) K04-7 and samples (d) K17-1, (e) K17-3, and (f) K17-5 synthesized from colloidal precursor suspensions aged four and seventeen days, respectively.
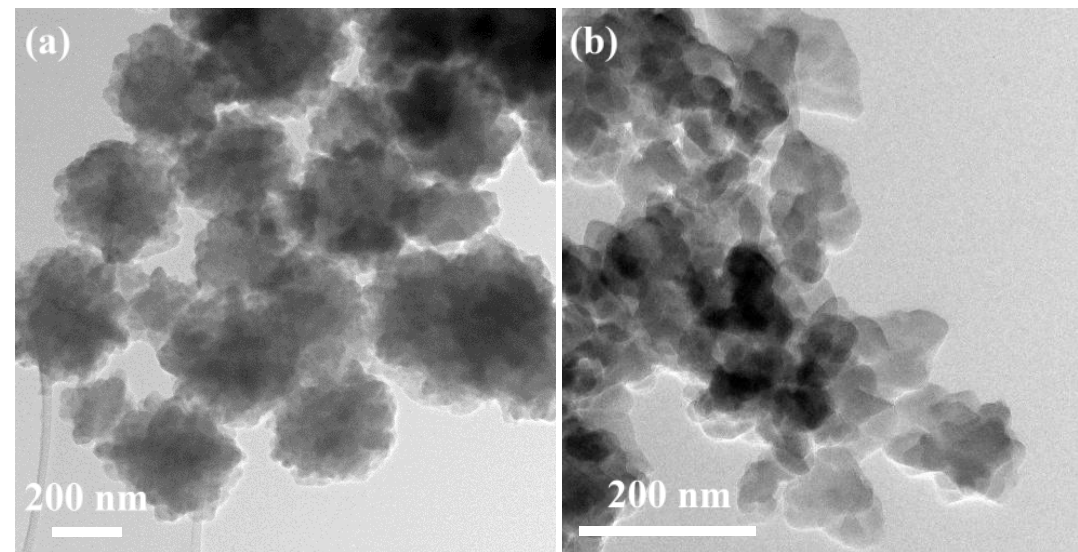

Figure 3. TEM images highlighting the morphology of fully crystalline nanosized CHA samples (a) K04-7 and (b) K17-3. 

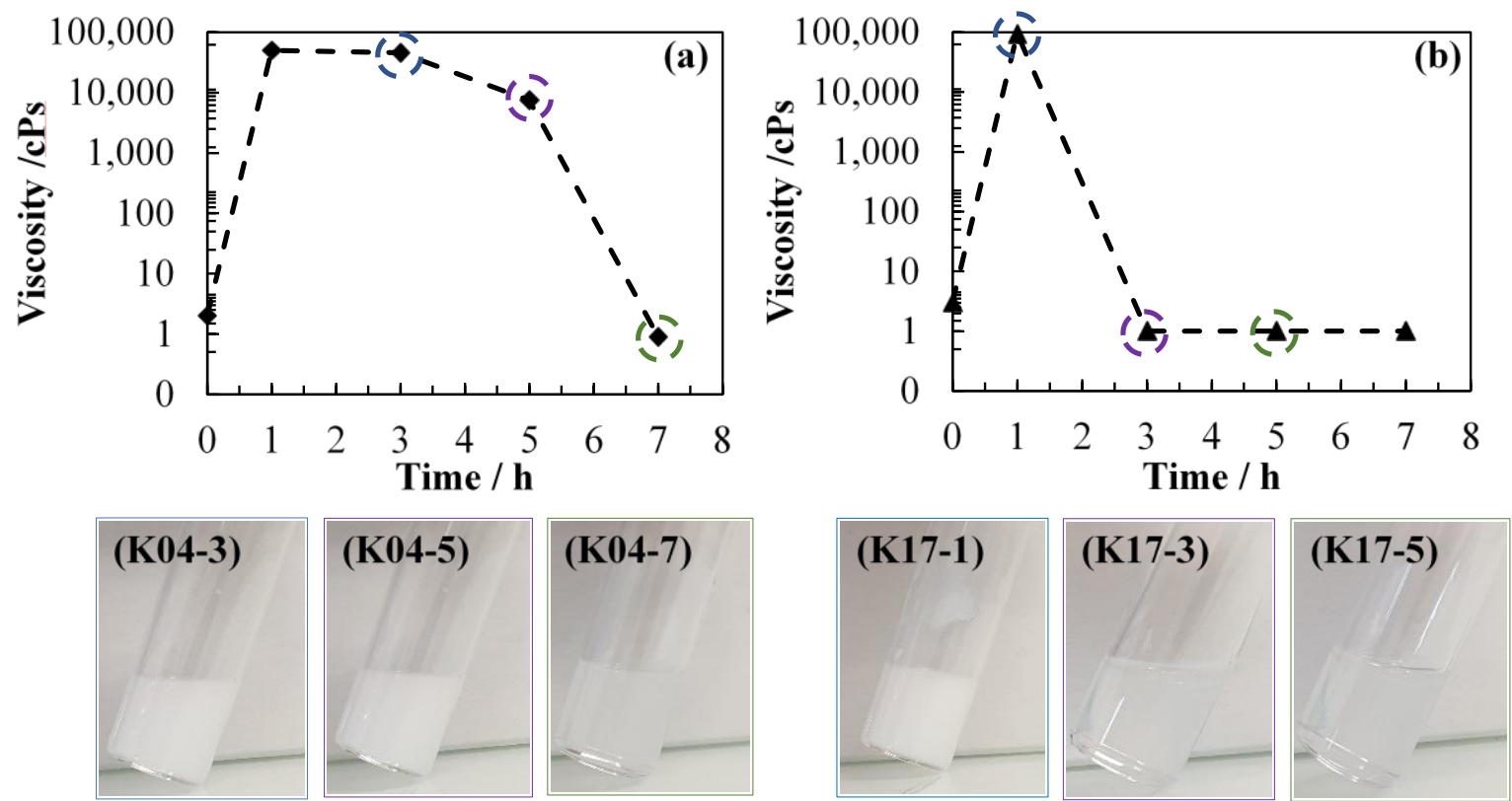

Figure 4. Top: viscosity changes of the colloidal precursor suspensions of samples from the series K04 and K17 at different times of hydrothermal treatment: (a) K04-0, K04-1, K04-3, K04-5, and K04-7, and (b) K17-0, K17-1, K17-3, K17-5, and K17-7. Bottom: pictures of the colloidal suspensions K04-3, K04-5, K04-7, K17-1, $\mathrm{K} 17-3$, and K17-5 

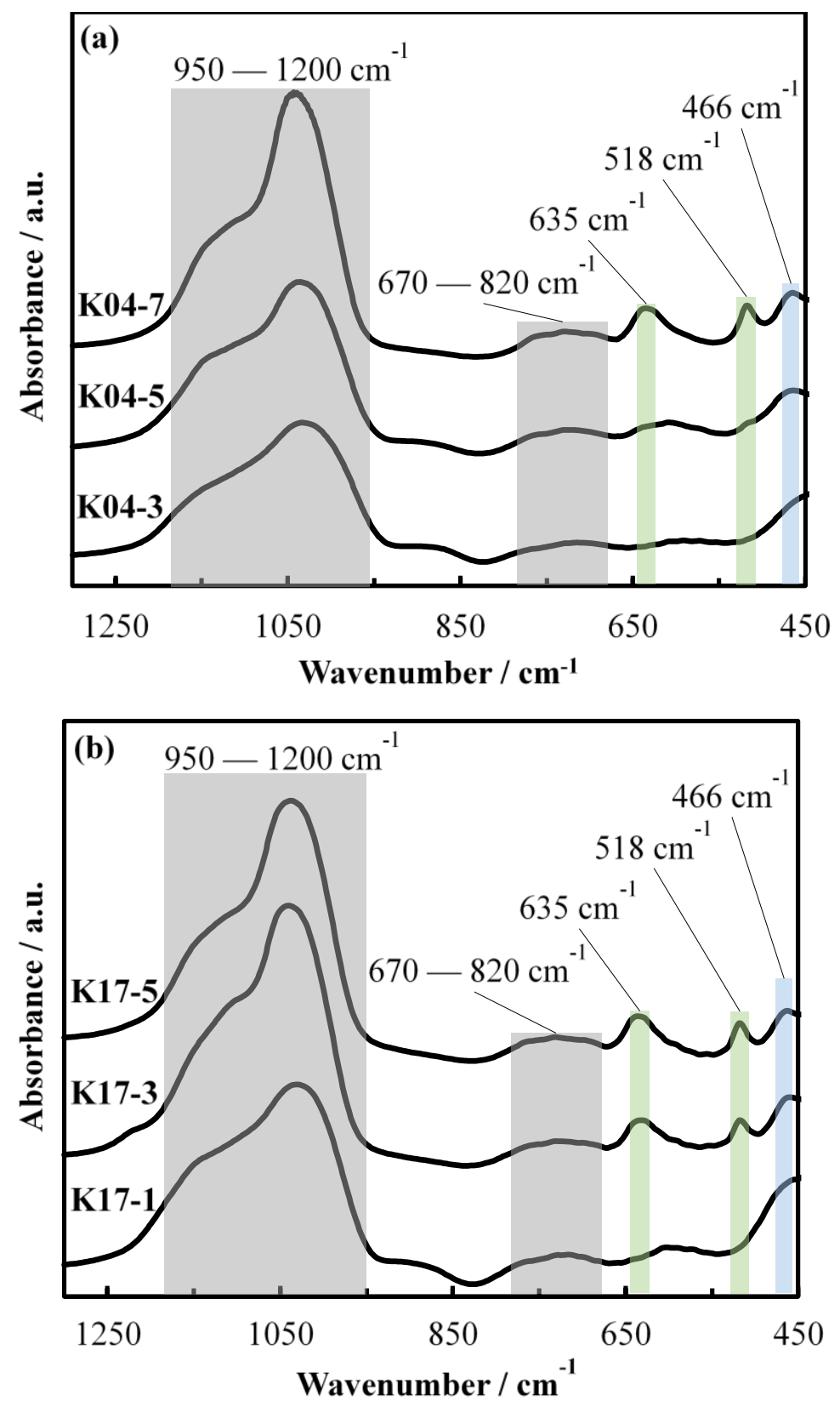

Figure 5. FTIR spectra of the nanosized CHA samples: (a) K04-3, K04-5, and K04-7 and (b) K17-1, K17-3, and K17-5 synthesized from colloidal precursor suspensions aged four and seventeen days, respectively. 

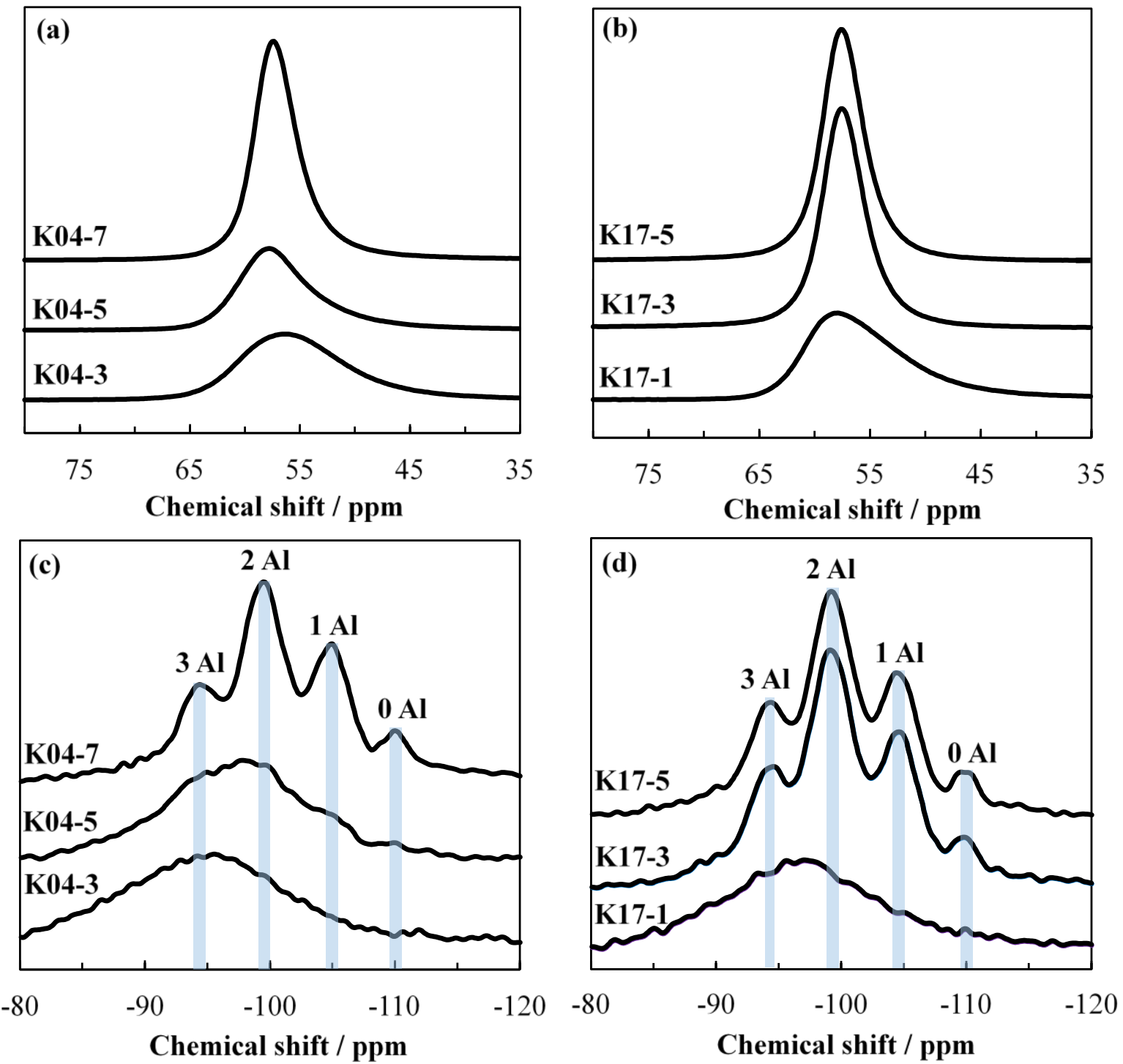

Figure 6. ${ }^{27} \mathrm{Al}$ MAS NMR spectra of the nanosized CHA samples (a) K04-3, K04-5, and K04-7, (b) K17-1, K17-3, and K17-5. ${ }^{29} \mathrm{Si}$ MAS NMR spectra of CHA samples (c) K04-3, K04-5, and K04-7, (d) K17-1, K17-3, and $\mathrm{K} 17-5$ of CHA samples. 

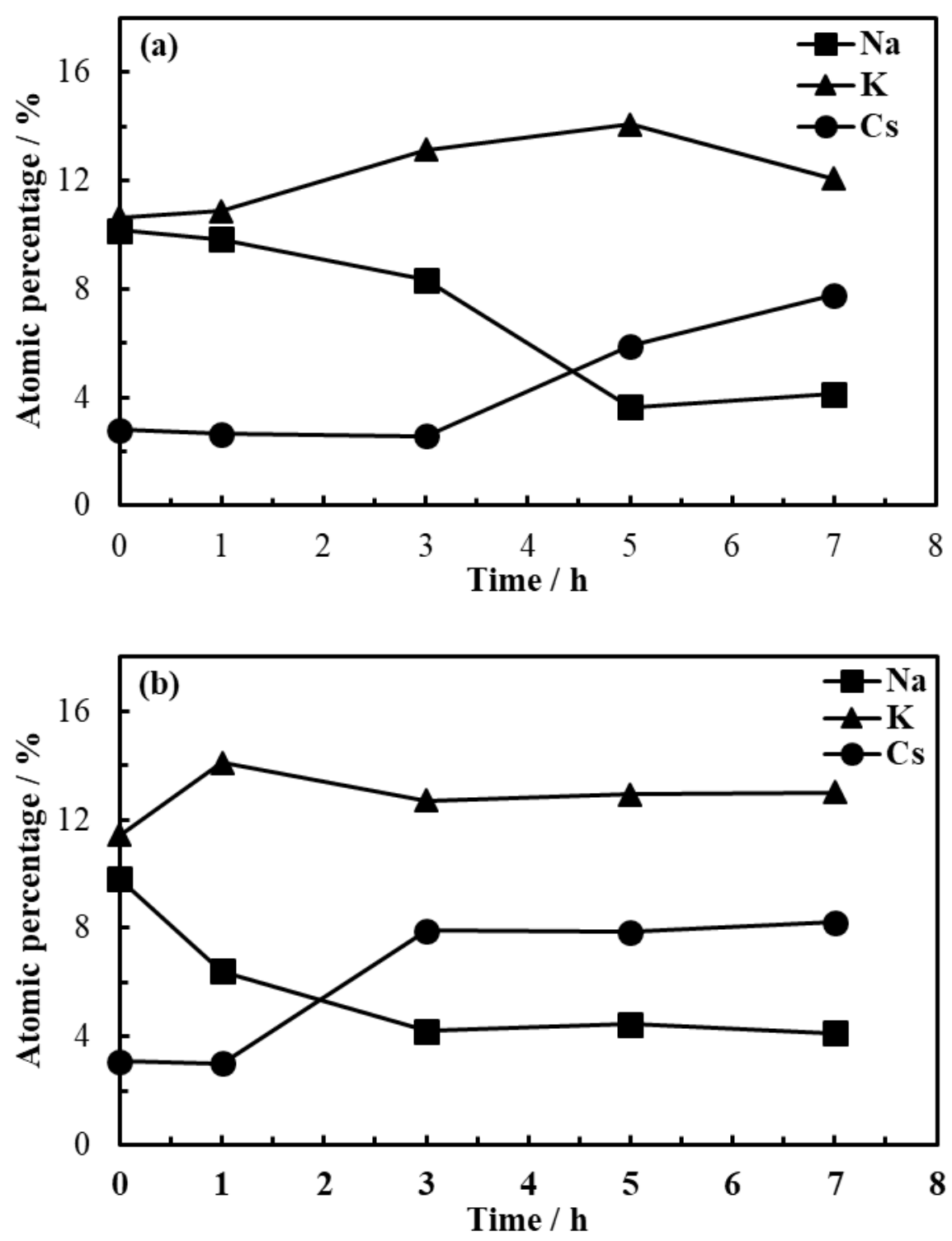

Figure 7. ICP-MS analysis revealing the change in the content of extra-framework cations $\left(\mathrm{Na}^{+}, \mathrm{K}^{+}, \mathrm{Cs}^{+}\right)$in the nanosized CHA series K04 and K17 at different times of hydrothermal treatment: (a) K04-0, K04-1, K043, K04-5, and K04-7, and (b) K17-0, K17-1, K17-3, K17-5, and K17-7. 
Table 1. Si/Al ratio of fully crystalline CHA samples (K04-7 and K17-3) based on ${ }^{29} \mathrm{Si}$ MAS NMR, ICP-MS, and EDX.

\begin{tabular}{cccc}
\hline Set & $\begin{array}{c}\text { Si/Al } \\
\left({ }^{29} \mathbf{S i ~ N M R}\right)\end{array}$ & $\begin{array}{c}\text { Si/Al } \\
(\text { ICP-MS })\end{array}$ & $\begin{array}{c}\text { Si/Al } \\
(\text { EDX })\end{array}$ \\
\hline K04-7 & 2.1 & 2.0 & 2.5 \\
\hline K17-3 & 2.1 & 1.9 & 2.5 \\
\hline
\end{tabular}




\section{Supporting information}

\section{The role of mixed alkali metal cations on the formation of nanosized CHA aluminosilicate zeolite from colloidal precursor suspension}

Sajjad Ghojavand ${ }^{\mathrm{a}}$, Edwin B. Clatworthy ${ }^{\mathrm{a}}$, Aurélie Vicente ${ }^{\mathrm{a}}$, Eddy Dib $^{\mathrm{a}}$, Valérie Ruaux ${ }^{\mathrm{a}}$, Maxime Debost $^{\mathrm{a}, \mathrm{b}}$, Jaafar El Fallah ${ }^{\mathrm{a}}$, Svetlana Mintova ${ }^{\mathrm{a}^{*}}$

${ }^{a}$ Normandie Université, ENSICAEN, UNICAEN, CNRS, Laboratoire Catalyse et Spectrochimie (LCS), 14000 Caen, France

${ }^{\mathrm{b}}$ Normandie Université, ENSICAEN, UNICAEN, CNRS, CRISMAT, 14000 Caen, France 
Table S1. Si/Al ratio of fully crystalline CHA samples (K04-7, K17-3, K17-5, and K17-7) based on ${ }^{29}$ Si MAS NMR, ICP-MS, and EDX analyses.

\begin{tabular}{cccc}
\hline Set & $\begin{array}{c}\text { Si/Al } \\
\left({ }^{\mathbf{2 9}} \mathbf{S i} \text { NMR }\right)\end{array}$ & $\begin{array}{c}\text { Si/Al } \\
(\text { ICP-MS })\end{array}$ & $\begin{array}{c}\text { Si/Al } \\
(\mathbf{E D X})\end{array}$ \\
\hline K04-7 & 2.1 & 2.0 & 2.5 \\
\hline K17-3 & 2.1 & 1.9 & 2.5 \\
\hline K17-5 & 2.1 & 1.9 & 2.4 \\
\hline K17-7 & 2.1 & 1.9 & 2.4 \\
\hline
\end{tabular}


Table S2. Parameters used to measure ${ }^{27} \mathrm{Al},{ }^{29} \mathrm{Si},{ }^{23} \mathrm{Na}$, and ${ }^{133} \mathrm{Cs}$ MAS NMR spectra

\begin{tabular}{ccccc}
\hline Nucleus & Recycle delay / s & Number of scans & Pulse angle & $\begin{array}{c}\text { Chemical shift } \\
\text { reference }\end{array}$ \\
\hline${ }^{27} \mathbf{A l}$ & 1 & 2048 & $\pi / 12$ & $\mathrm{Al}\left(\mathrm{NO}_{3}\right)_{3}$ \\
\hline${ }^{29} \mathbf{S i}$ & 15 & 3072 & $\pi / 6$ & $\mathrm{Si}\left(\mathrm{CH}_{3}\right)_{4}$ \\
\hline${ }^{23} \mathbf{N a}$ & 1 & 4096 & $\pi / 12$ & $\mathrm{NaCl}$ \\
\hline${ }^{133} \mathbf{C s}$ & 2 & 4096 & $\pi / 12$ & $\mathrm{CsCl}$ \\
\hline
\end{tabular}




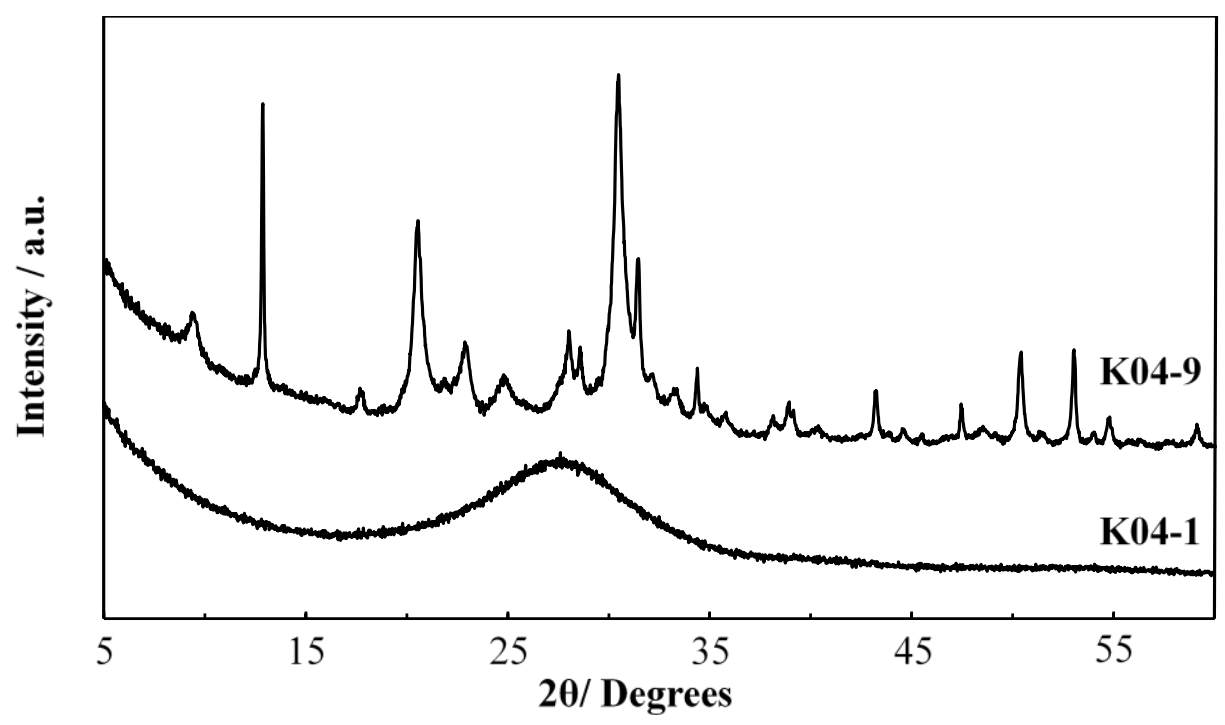

Figure S1. XRD patterns of the nanosized CHA from series K04: K04-1, and K04-9 samples. 


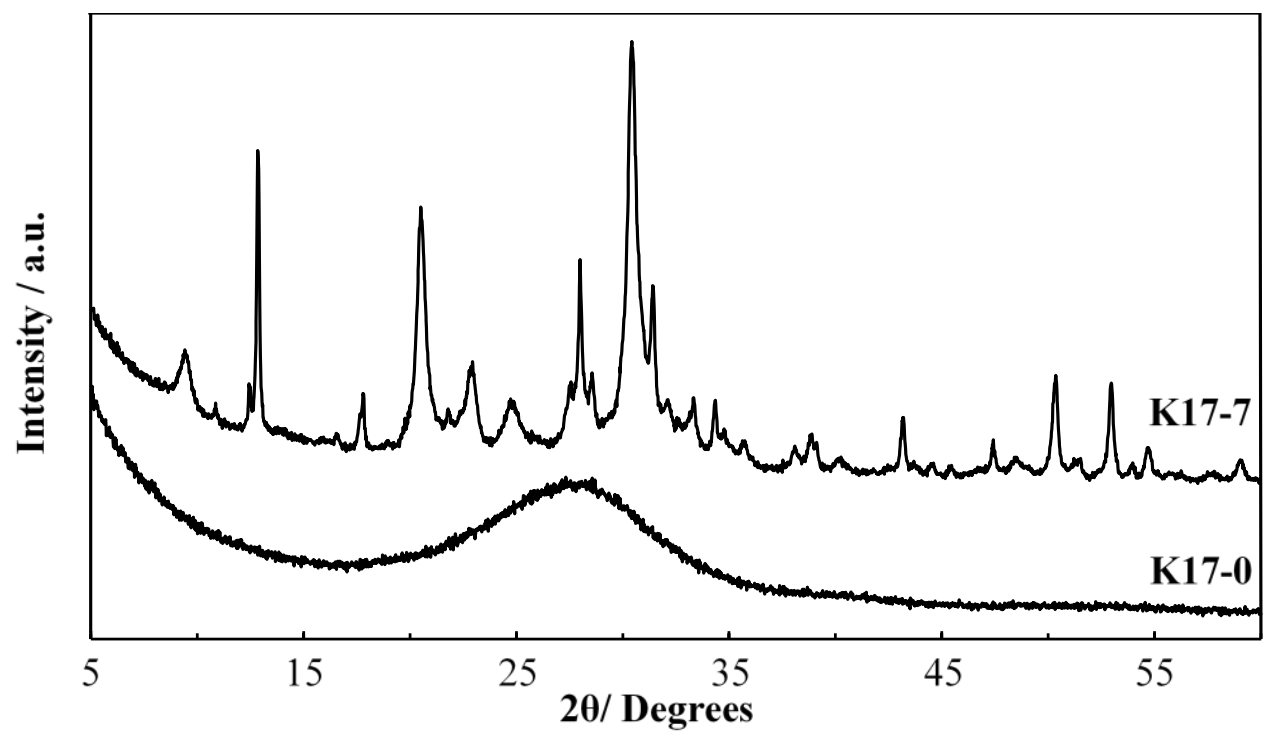

Figure S2. XRD patterns of the nanosized CHA from series K17: K17-0, and K17-7 samples. 

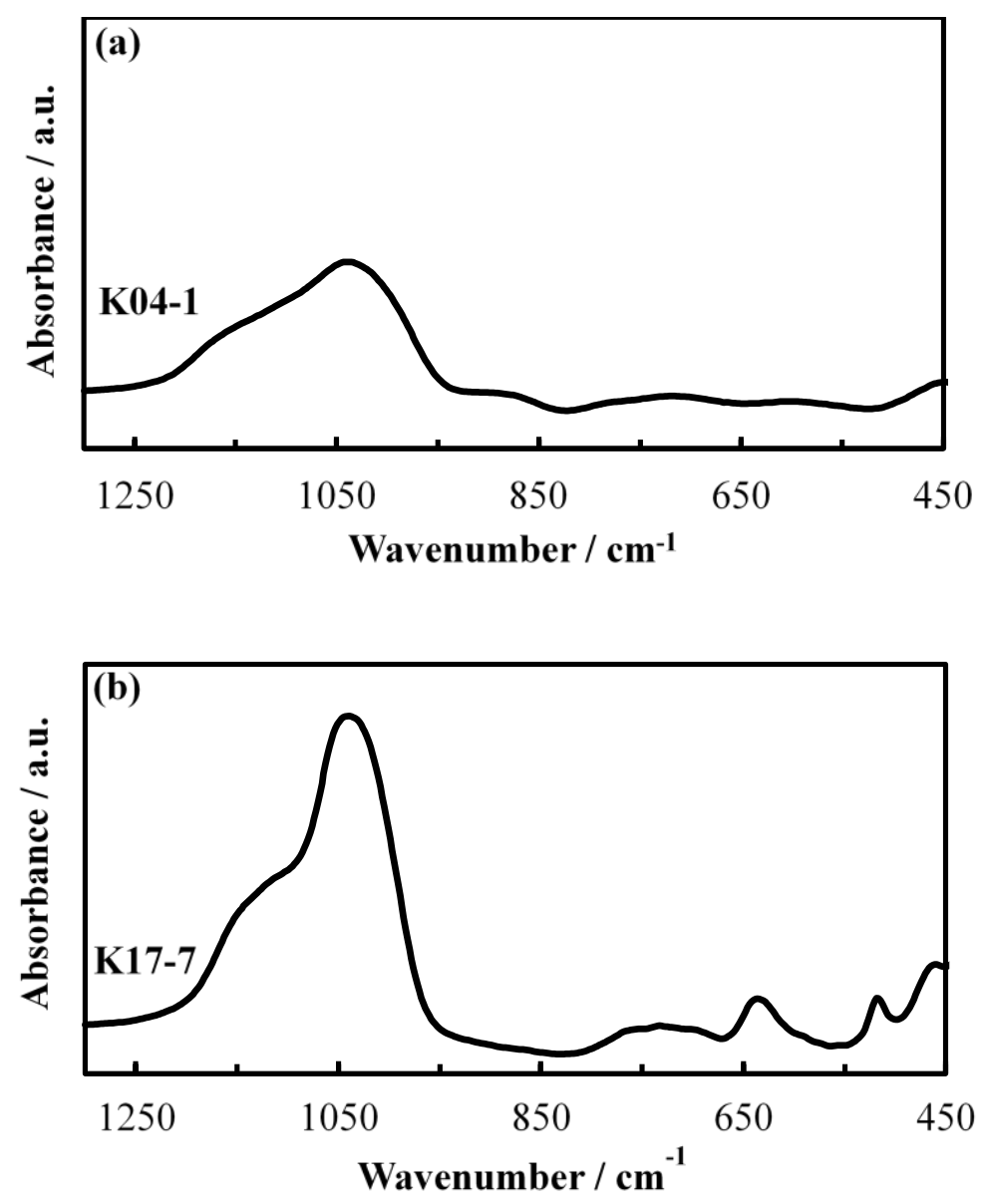

Figure S3. FTIR spectra of the nanosized CHA (a) K04-1 and (b) K17-7 samples. 

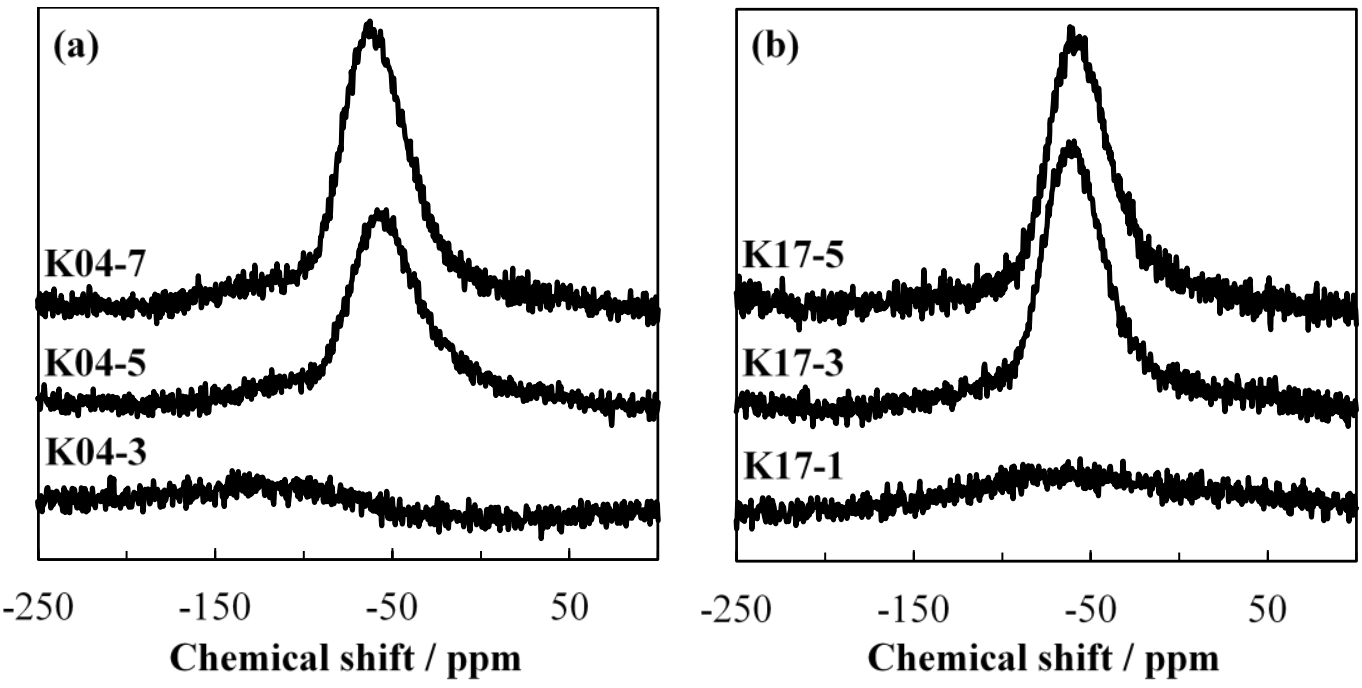

Figure S4. ${ }^{133}$ Cs MAS NMR spectra of the nanosized CHA from (a) series K04: K04-3, K04-5, K04-7, and (b) series K17: K17-1, and K17-3 CHA, respectively. 

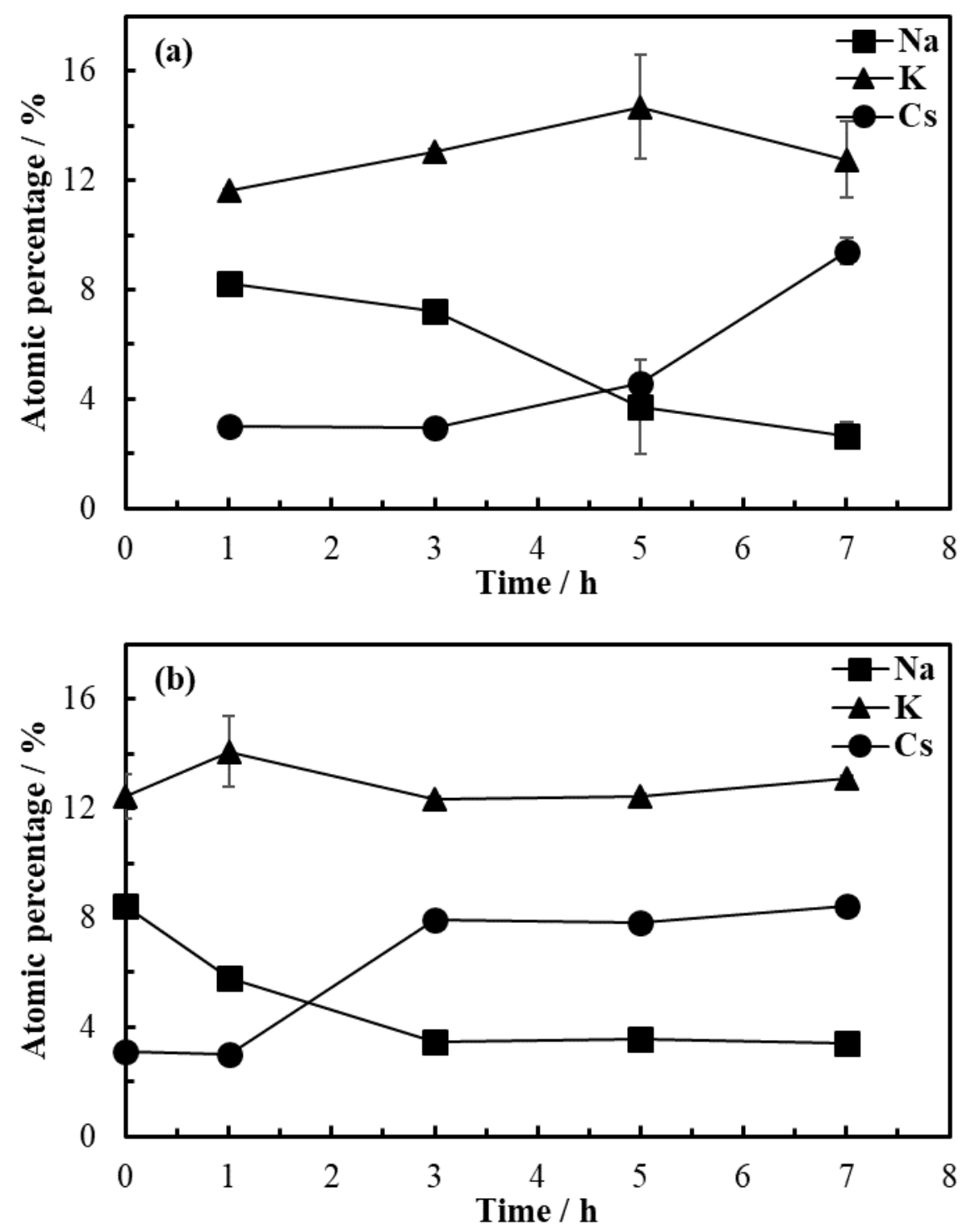

Figure S5. EDX analysis of the nanosized CHA representing the change in the content of extra-framework cations $\left(\mathrm{Na}^{+}, \mathrm{K}^{+}, \mathrm{Cs}^{+}\right)$in (a) series K04: K04-0, K04-1, K04-3, K04-5, and K04-7 and (b) series K17: K17-0, $\mathrm{K} 17-1, \mathrm{~K} 17-3$, K17-5, and K17-7; results are averaged for three measurements and the standard deviations are represented by error bars. 

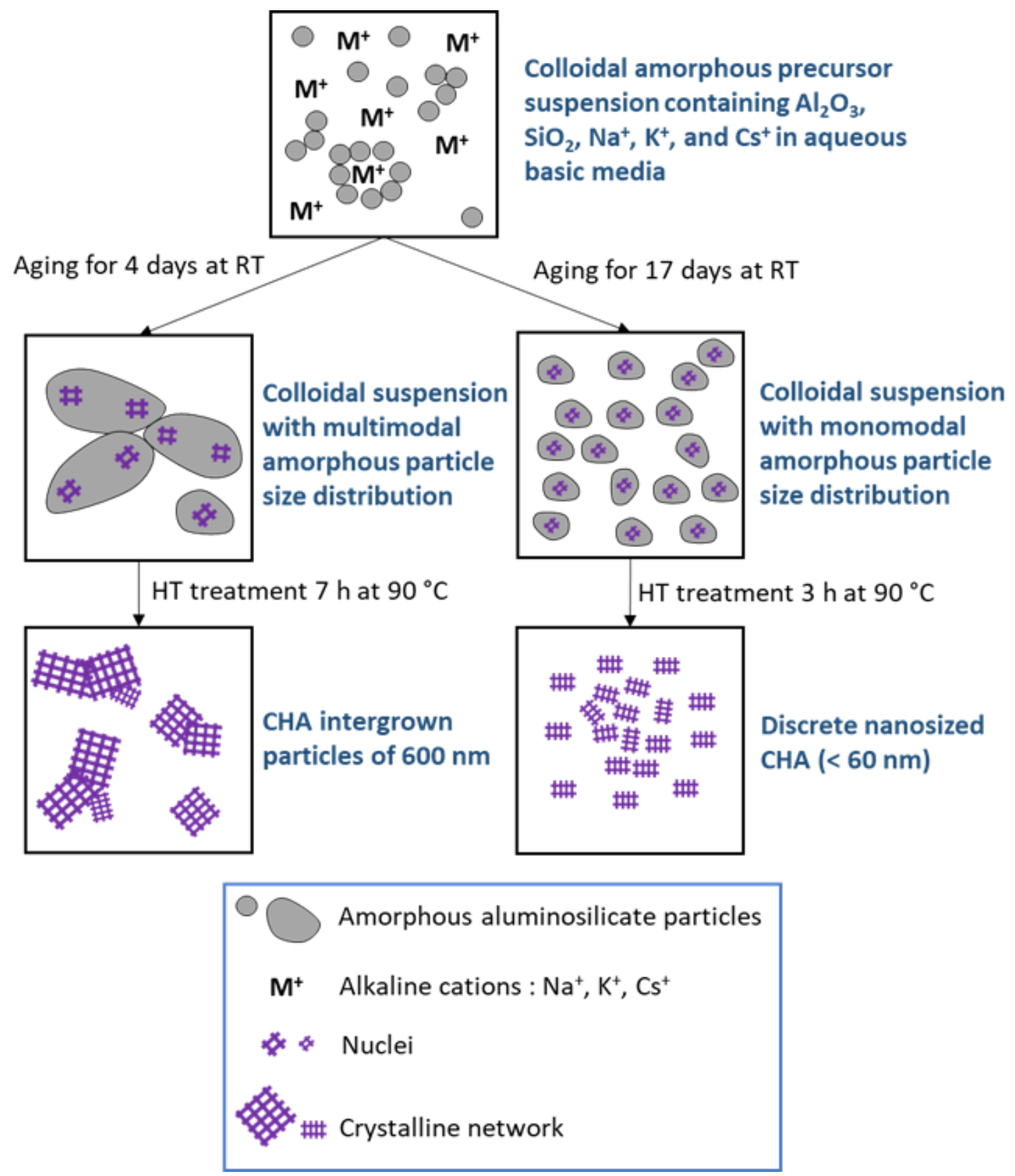

Scheme S1. Schematic illustration of the proposed crystallization pathway of nanosized CHA zeolite in colloidal precursor suspensions for series K04 and K17. 